\title{
Erratum to: A new approach to the design of RC structures based on concrete mix characteristic length
}

\author{
B. L. Karihaloo
}

Published online: 1 April 2015

(C) Springer Science+Business Media Dordrecht 2015

\section{Erratum to: Int J Fract (2015) 191:147-165 \\ DOI 10.1007/s10704-015-0009-2}

Due to an unfortunate turn of events this article was published with an erroneous version of Table 1. Please find in this erratum the correct version of Table 1 that should be regarded by the reader as the final version.

Table 1 Material properties

\begin{tabular}{lllll}
\hline $\begin{array}{l}\text { Concrete mix } \\
\text { designation }\end{array}$ & $\begin{array}{l}\text { Static modulus of } \\
\text { elasticity } E(\mathrm{GPa})\end{array}$ & $\begin{array}{l}\text { True specific } \\
\text { fracture energy } \\
G_{F}(\mathrm{~N} / \mathrm{m})\end{array}$ & $\begin{array}{l}\text { Tensile splitting } \\
\text { strength } f_{t}(\mathrm{MPa})\end{array}$ & $\begin{array}{l}\text { Characteristic } \\
\text { length } l_{c h}(\mathrm{~mm})\end{array}$ \\
\hline C40-C50 & $30-40$ & $90-125$ & $3.5-4.0$ & $225-305$ \\
C100-C110 & $45-52$ & $60-80$ & $6.5-7.5$ & $55-85$ \\
C100-C110+0.18-0.20\% & $45.5-52.5$ & $450-500$ & $7.8-8.4$ & $335-375$ \\
by vol steel fibre & & & & 470 \\
C85+0.2\% SF & 41.2 & 546 & 8.7 & 750 \\
C85+0.5\% SF & 41.6 & 1361 & 10.1 & 970 \\
C85+0.75 \% SF & 41.8 & 2448 & & \\
\hline
\end{tabular}

The online version of the original article can be found under doi:10.1007/s10704-015-0009-2.

B. L. Karihaloo ( $\square)$

School of Engineering, Cardiff University,

Cardiff CF24 3AA, UK

e-mail: KarihalooB@Cardiff.ac.uk 